'Matrona, Magister en Salud Pública. Hospital San Juan de Dios de La Serena. La Serena, Chile.

${ }^{2}$ Médico Especialista en Salud Pública. Magister en Salud Pública. PhDœ International Public Health. Departamento de Salud Pública, Facultad de Medicina, Universidad Católica del Norte.Coquimbo, Chile.

Recibido el 6 de noviembre de 2019, aceptado el 10 de agosto de 2020.

Correspondencia a: Muriel Ramírez-Santana Calle Larrondo 1281, Coquimbo, Chile. mramirezs@ucn.cl

Material suplementario en versión digital de la Revista Médica de Chile en: Http://revmedchile.org/ material-suplementario

\section{Epidemiología de la sífilis con enfoque territorial: caso del Hospital de La Serena, años 2015-2017}

\author{
ANDREA CAVERO TARDONES ${ }^{1}$, MURIEL RAMÍREZ-SANTANA ${ }^{2}$
}

\section{Epidemiological features of syphilis diagnosed at a clinic of sexually transmitted diseases}

Background: Syphilis continues to be a common sexually transmitted disease. Aim: To describe the epidemiologic features of patients with syphilis consulting in a Chilean sexually transmitted diseases clinic. Material and Methods: Review of medical records of patients with positive serology for syphilis, who attended a unit of sexually transmitted diseases between 2015 and 2017. Results: Of 266 cases, $61 \%$ were male and $74 \%$ were aged between 20 and 49 years. The highest incidence was observed in males aged between 20 and 30 years. Eighty five percent of cases were diagnosed in latent phases, $10 \%$ of the cases had a co-infection with HIV and 18\%, a history of other sexually transmitted disease. The rate of coinfection was significantly higher in men than in women (78 and $28 \%$, respectively; $p<0.01)$. Only $6.4 \%$ reported always using a condom. Men used it with higher frequency than women ( 72 and $28 \%$ respectively; $p<0.01$ ). A low adherence to treatment and faulty follow-up and treatment of sexual contacts was observed. Twenty five percent of women diagnosed with syphilis were pregnant and $27 \%$ of them had a premature birth, with one neonatal death. Conclusions: A high incidence of syphilis was observed, especially in men of younger ages. The diagnosis is delayed, the treatment of sexual contacts and the use of condoms are uncommon. Although the treatment of pregnant women is appropriated, some perinatal complications are observed.

(Rev Med Chile 2020; 148: 956-962)

Key words: Pregnancy Complications; Syphilis; Sexually Transmitted Diseases; Unsafe Sex.

\section{L} a sífilis es producida por la bacteria Treponema Pallidum, observándose en población sexualmente activa como una Infección de Transmisión Sexual (ITS) de alta frecuencia a nivel mundial y nacional ${ }^{1-4}$. Se presenta con una variedad de cuadros clínicos: sífilis primaria, sífilis secundaria, latente precoz, latente tardía y sífilis terciaria ${ }^{5}$. El cuadro de sífilis congénita, origina secuelas en recién nacidos, además de prematurez y muerte neonatal ${ }^{6,7}$. A pesar de que el tratamiento con penicilina es eficaz y de bajo costo ${ }^{5}$, sigue siendo un problema de salud pública, con implicancias sociales y económicas ${ }^{3,8}$.
La sífilis es una enfermedad de notificación obligatoria (ENO) en Chile y en el mundo ${ }^{8-10}$; existiendo programas de control y prevención con algoritmos de pesquisa, diagnóstico y tratamiento precoz; además de estrategias definidas para la prevención de la transmisión vertical ${ }^{3,8,9,11-14}$, así como de búsqueda y manejo de contactos sexuales ${ }^{2,9}$.

La evidencia muestra que presentar una ITS aumenta el riesgo de adquirir otras, entre ellas el Virus de Inmunodeficiencia Humana (VIH) ${ }^{2,15}$. La epidemia de VIH y los avances en su tratamiento han traído consigo cambios en la epidemiología de otras ITS, incluida la sífilis ${ }^{3}$. En países europeos 
se ha descrito recientemente que la morbilidad por sífilis afecta mayormente a hombres jóvenes, pertenecientes al grupo de hombres que tienen sexo con hombres $(\mathrm{HSH})^{8}$. Aparentemente esta misma situación se está presentando en nuestro país ${ }^{16}$. En este contexto, conocer las características de la morbilidad por sífilis en un territorio, es imprescindible para orientar las estrategias de control de la enfermedad. El estudio tuvo como objetivo conocer la epidemiología de la sífilis en el área de influencia del Hospital de La Serena, caracterizando los casos según variables demográficas, clínicas y de salud sexual y reproductiva.

\section{Material y Métodos}

Se realizó un estudio descriptivo, transversal y retrospectivo, utilizando el registro de usuarios mayores de 15 años, con serología reactiva para Sífilis, atendidos en la Unidad de Atención y Control de Salud Sexual (UNACESS) del Hospital San Juan de Dios de La Serena, de tres años (20152017). La población universo fueron beneficiarios del Fondo Nacional de Salud (FONASA), mayores de 15 años, de las comunas que derivan sus pacientes a dicho hospital (La Higuera, La Serena, Paihuano y Vicuña). Los datos de población para el denominador de las tasas fueron extraídos de la página web del Instituto Nacional de Estadísticas, INE ${ }^{17}$ y ajustados a $78 \%$ correspondiente a población FONASA ${ }^{18}$.

Se revisó manualmente la información de todos los tarjetones de atención archivados en la UNACESS, transcribiendo los datos a planilla computacional. Las variables estudiadas fueron edad, sexo, comuna, antecedentes de ITS y su etiología, co-infección con VIH, etapa de la sífilis, adherencia al tratamiento, información sobre contactos, tipo de relación de pareja, orientación sexual y uso de preservativo. La información de resultados perinatales se obtuvo de los registros de partos de maternidad, validados con la información del Departamento de Estadísticas e Información de Salud del Ministerio de Salud de Chile (DEIS). Se excluyeron variables sensibles de los casos (nombre, RUT y dirección). El manejo de la información se realizó respetando las normas éticas concordantes con la Declaración de Helsinki y los principios de confidencialidad, sin identificación alguna del usuario/a. Se contó con la autorización del Director del establecimiento.

Toda la información fue ingresada en dos matrices de datos en el programa Microsoft Excel ${ }^{\circledR}$. La primera matriz permitió estimar las tasas de incidencia del territorio en estudio, según año, sexo y grupos quinquenales de edad. La segunda matriz facilitó la caracterización de los casos, describiendo las variables en cifras absolutas y relativas (porcentajes). Se evaluó la relación del sexo con la presencia de co-infección con otras ITS y con el uso de preservativo; a través de cálculo de Chi cuadrado de Pearson, con 95\% de confianza, utilizando el programa SPSS $^{\circledR}$-IBM v26.

\section{Resultados}

Se obtuvo un total de 266 registros para el período de tres años; de los cuales 161 casos fueron de sexo masculino (60\%) y $105(40 \%)$, de sexo femenino; de ellas 26 eran gestantes $(24,7 \%)$.

La Figura 1 presenta el número de casos incidentes según sexo y grupos de edad. Las tasas de incidencia estimadas se muestran en la Tabla suplementaria 1 . Se observa tasas globales entre 32 y 55 por cien mil habitantes, mayores en hombres que en mujeres. El año 2015 la distribución por edad fue homogénea, con tasas sobre 50 por cien mil habitantes y concentradas entre los 20 y 49 años. Luego, en los años siguientes, los grupos edad entre 20 y 34 años reúnen tasas superiores a 100 por cien mil habitantes, principalmente en hombres. El mayor cambio se observa en el grupo de hombres de 20 a 30 años, en el cual la tasa de incidencia se cuadruplica desde el año 2015 al 2017 (de 43,5 a 177,3 por 100.000 habitantes). Se evidencia disminución de las tasas en edades sobre 40 años. La descripción según comunas se presenta como material suplementario (Tabla suplementaria 2).

La Tabla 1 presenta las características de los casos de sífilis. El diagnóstico se originó principalmente en los controles de salud $(80 \%)$ y menormente en controles ginecológicos o donantes (7,5\% y 9,4\% respectivamente). La mayoría de los casos se diagnosticaron en etapas latentes (precoz $45,5 \%$ y tardía $39,1 \%$ ); $8,3 \%$ en etapa secundaria; solo $6,4 \%$ fueron sífilis primaria. Un $72 \%$ de los pacientes adhirió a sus controles y $28 \%$ quedaron clasificados como inasistentes. Solo se realizó tratamiento de los contactos en un tercio de los casos. 


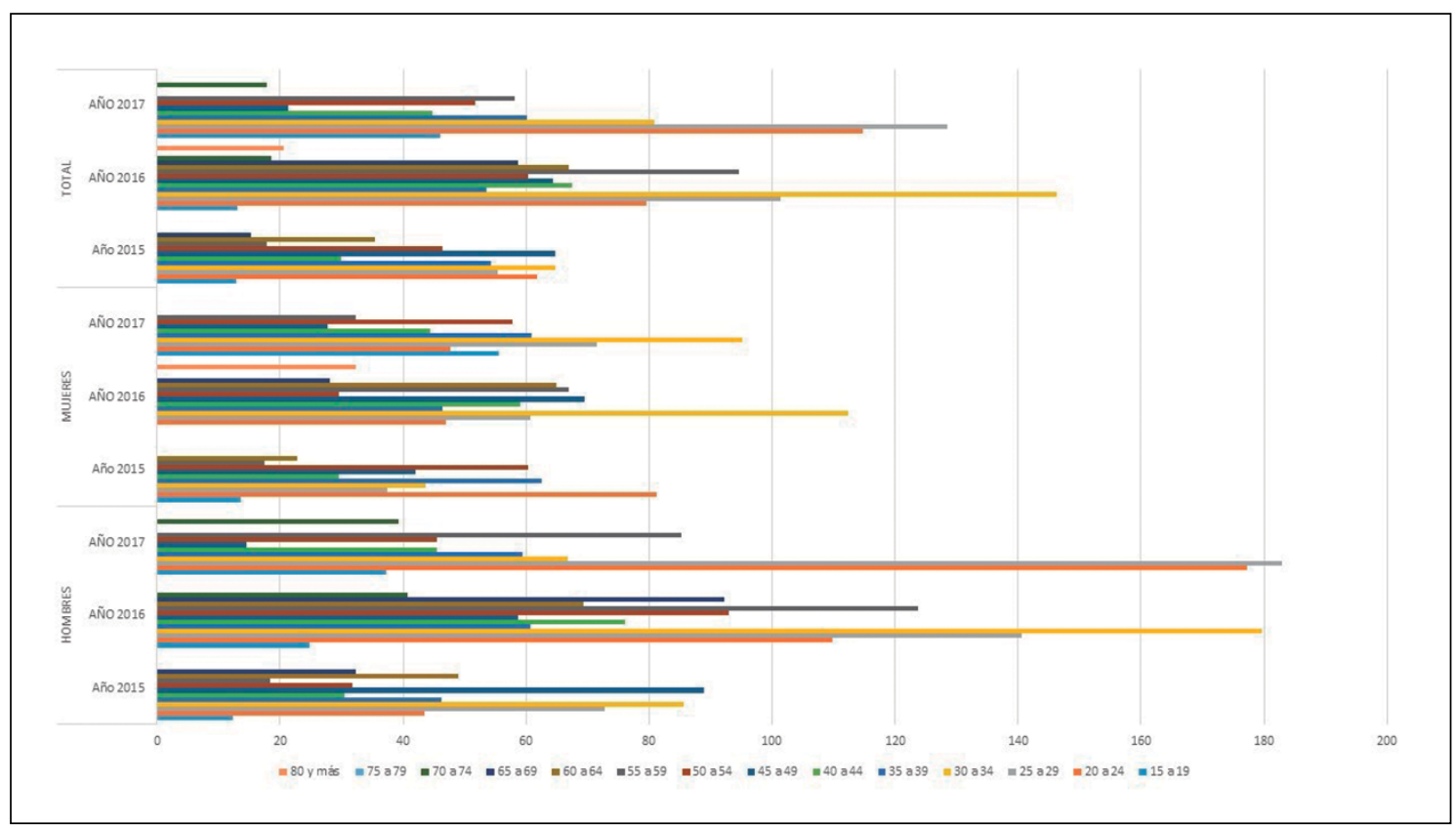

Figura 1. Tasas de incidencia de sífilis por cien mil habitantes en área de influencia del Hospital de La Serena, según sexo y grupos de edad, años 2015 a 2017.

Cerca de 10\% (26 casos) tuvieron co-infección con VIH y casi el 20\%, antecedentes de otra ITS. $\mathrm{El}$ antecedente de ITS/VIH fue significativamente mayor en hombres que en mujeres $(72,2 \%$ versus $22,4 \%$, respectivamente $\mathrm{p}<0,001$, datos no mostrados). En relación a la orientación sexual, la mayoría informó ser heterosexual $(73,7 \%)$ y un $16,9 \%$ declaró ser homosexual. Cerca de la mitad (48\%) informó tener una relación estable y sexualmente activa, $22 \%$ dijo tener relaciones ocasionales y $25 \%$ no informó actividad sexual. Solo $6,4 \%$ declaró usar preservativo "siempre"; cerca de $40 \%$ lo usa "a veces"; $36 \%$ "no usa" y $18 \%$ no informa si usa o no. Se encontró diferencias significativas en el uso de condón entre hombres y mujeres. El uso es mayor en hombre $(72,2 \%)$ que en mujeres (27,8\%). El 26,7\% de los hombres no usa condón, mientras que $46,5 \%$ de las mujeres no los usa ( $\mathrm{p}=0,002$; datos no mostrados).

En los tres años de estudio se observaron 26 casos de sífilis gestacional (9,7\% del total de casos y $24,7 \%$ de las mujeres). Los casos de gestantes se concentraron entre los 20 a 39 años, presentándose un caso en adolescente (año 2017) y pocos casos en edades entre 40 y 44 años. La Tabla 2 muestra que la mayoría de los casos en gestantes se diagnosticó en el primer (46\%) o segundo trimestre (35\%). Cerca de 90\% recibió adecuadamente su tratamiento; sin embargo, solo $65 \%$ de las parejas fueron debidamente tratadas. El 70\% de los casos tuvo resultados perinatales normales. El principal resultado perinatal desfavorable fue la prematurez ( $27 \%$ de los casos) y hubo una muerte neonatal $(4 \%)$.

\section{Discusión}

El estudio detalla las características de morbilidad de la sífilis en el territorio estudiado. Los registros de enfermedades de notificación obligatoria (ENO) carecen de variables de interés como: etapa del diagnóstico, co-infección, adherencia al tratamiento, información de contactos, conducta sexual y uso de condón ${ }^{19}$. Así mismo, es un aporte al describir el perfil de la sífilis gestacional, para la cual no existe un análisis sistemático de información que sea publicado. De hecho, los Boletines Epidemiológicos solo reportan la morbilidad nacional (casos notificados y de gestantes en ge- 
Tabla 1. Caracterización de los casos de sífilis atendidos en el Hospital de La Serena, período 2015-2107

\begin{tabular}{|c|c|c|}
\hline Variable & $\mathbf{n}$ & $\begin{array}{c}\text { Frecuencia } \\
\text { relativa } \\
(\%)\end{array}$ \\
\hline \multicolumn{3}{|l|}{ Origen solicitud de examen } \\
\hline Control de salud & 213 & 80,0 \\
\hline Control ginecológico & 25 & 9,4 \\
\hline Donante & 20 & 7,5 \\
\hline Recinto carcelario & 5 & 1,9 \\
\hline Hallazgo hospitalario & 3 & 1,1 \\
\hline Total & 266 & 100 \\
\hline \multicolumn{3}{|l|}{ Clasificación clínica de los casos } \\
\hline Latente precoz & 121 & 45,5 \\
\hline Latente tardía & 104 & 39,1 \\
\hline Primaria & 17 & 6,4 \\
\hline Secundaria & 22 & 8,3 \\
\hline Neurosífilis & 2 & 0,8 \\
\hline Total & 266 & 100 \\
\hline \multicolumn{3}{|l|}{ Información de contactos } \\
\hline Contactos tratados & 89 & 33,4 \\
\hline Contactos sin tratamiento & 79 & 29,7 \\
\hline Sin información de contactos & 98 & 36,8 \\
\hline Total & 266 & 100 \\
\hline \multicolumn{3}{|l|}{ Adherencia a controles } \\
\hline Con adherencia & 191 & 71,8 \\
\hline Inasistentes & 75 & 28,2 \\
\hline Total & 266 & 100 \\
\hline \multicolumn{3}{|l|}{ Antecedentes de ITS } \\
\hline Co-infección VIH & 26 & 9,8 \\
\hline Con antecedentes otras (no VIH)* & 49 & 18,4 \\
\hline Sin antecedentes & 191 & 71,8 \\
\hline Total & 266 & 100 \\
\hline \multicolumn{3}{|l|}{ Actividad sexual declarada } \\
\hline Activa en relación estable & 127 & 47,7 \\
\hline Activa relaciones casuales & 59 & 22,2 \\
\hline No informado & 68 & 25,6 \\
\hline Sin actividad & 12 & 4,5 \\
\hline Total & 266 & 100 \\
\hline \multicolumn{3}{|l|}{ Orientación sexual declarada } \\
\hline Heterosexual & 196 & 73,7 \\
\hline Homosexual & 45 & 16,9 \\
\hline Bisexual & 4 & 1,5 \\
\hline Transexual & 1 & 0,4 \\
\hline No informado & 20 & 7,5 \\
\hline Total & 266 & 100 \\
\hline \multicolumn{3}{|l|}{ Uso de preservativo } \\
\hline Siempre & 17 & 6,4 \\
\hline A veces & 106 & 39,8 \\
\hline No usa & 95 & 35,7 \\
\hline No informa & 48 & 18,0 \\
\hline Total & 266 & 100 \\
\hline
\end{tabular}

* $54 \%$ portadores de Hepatitis B, 20\% condiloma, 20\% sífilis previa, $12 \%$ ureaplasma y $8 \%$ gonorrea.
Tabla 2. Distribución de frecuencia relativa de sífilis en gestantes, según variables perinatales de los casos atendidos en el Hospital de La Serena, período 2015-2107 (n = 26)

\begin{tabular}{|c|c|c|}
\hline Variables & $n$ & $\begin{array}{c}\text { Frecuencia } \\
\text { relativa } \\
(\%)\end{array}$ \\
\hline $\begin{array}{l}\text { Edad gestacional } \\
\text { Primer trimestre } \\
\text { Segundo trimestre } \\
\text { Tercer trimestre } \\
\text { Parto }\end{array}$ & $\begin{array}{r}12 \\
9 \\
3 \\
2\end{array}$ & $\begin{array}{r}46,2 \\
34,6 \\
11,5 \\
7,7\end{array}$ \\
\hline $\begin{array}{l}\text { Paridad } \\
\text { Primigesta } \\
\text { Multípara }\end{array}$ & $\begin{array}{r}7 \\
19\end{array}$ & $\begin{array}{l}26,9 \\
73,1\end{array}$ \\
\hline $\begin{array}{l}\text { Tipo de tratamiento gestante } \\
\text { Adecuado } \\
\text { Inadecuado } \\
\text { Sin tratamiento }\end{array}$ & $\begin{array}{r}23 \\
1 \\
2\end{array}$ & $\begin{array}{r}88,5 \\
3,8 \\
7,7\end{array}$ \\
\hline $\begin{array}{l}\text { Tipo de tratamiento pareja } \\
\text { Adecuado } \\
\text { Inadecuado } \\
\text { Sin tratamiento }\end{array}$ & $\begin{array}{r}17 \\
7 \\
2\end{array}$ & $\begin{array}{r}65,4 \\
26,9 \\
7,7\end{array}$ \\
\hline $\begin{array}{l}\text { Resultado perinatal } \\
\text { Aborto } \\
\text { Prematurez } \\
\text { Muerte fetal } \\
\text { Muerte neonatal } \\
\text { Malformaciones } \\
\text { Sin alteraciones }\end{array}$ & $\begin{array}{r}0 \\
7 \\
0 \\
1 \\
0 \\
18\end{array}$ & $\begin{array}{c}0 \\
26,9 \\
0 \\
3,8 \\
0 \\
69,2\end{array}$ \\
\hline
\end{tabular}

neral); detallando su distribución según edad, sexo y regiones ${ }^{20}$. Recientemente se ha incorporado la variable nacionalidad en los registros oficiales, que permitirá identificar si la condición de migrante es un factor de mayor riesgo, como está descrito internacionalmente ${ }^{3}$. Esta publicación tiene la limitación de no consignar esta variable, dado que no existía en los registros de los años estudiados.

Las tasas globales de incidencia estimada varían entre 32 y 54 casos por cien mil habitantes; siendo equivalentes a la tasa descrita para Chile el año 2017, que bordea los 35 casos por cien mil habitantes ${ }^{20}$. La incidencia estimada se asemeja a los 44 casos por cien mil habitantes que presentó la región de Coquimbo el año $2017^{20}$. Las comunas con mayor riesgo de enfermar fueron La Higuera y La Serena. El estudio, al ser de tres años, no permite predecir una tendencia de la incidencia. Lo cual sí es posible de observar a nivel país, que 
presenta una preocupante tendencia ascendente de la incidencia, en los últimos 5 años $^{20}$. Puede considerarse una limitación del estudio no incluir casos potencialmente diagnosticados y tratados en centros de atención primaria u hospital de baja complejidad, sin ser derivados al Hospital de La Serena. Sin embargo, las tasas observadas coinciden con las regionales y nacionales, apoyando los resultados del estudio.

Una observación de interés es el desplazamiento de la incidencia hacia varones jóvenes, con tasas superiores a 100 por cien mil habitantes en las edades entre 20 y 30 años, en los dos últimos años estudiados. Estas tasas duplican las nacionales de los mismos grupos de edad ${ }^{16}$. Por otra parte, en el año 2017, se observa un aumento de la incidencia en adolescentes, que afecta tanto a hombres como a mujeres (una gestante en el año 2017). El mismo perfil se informa en reportes nacionales ${ }^{16,20} \mathrm{e}$ internacionales. Cáceres comunica en un estudio que, a partir del año 2016, aumenta la morbilidad en adolescentes chilenos ${ }^{16}$; así mismo, se observa preponderancia de la incidencia en hombres entre 20 y 30 años en el último Boletín Epidemiológico nacional ${ }^{20}$. El reporte anual de sífilis de la Unión Europea 2017 describe claramente este cambio en el perfil epidemiológico de la enfermedad ${ }^{8}$, con mayor riesgo de enfermar en hombres jóvenes. No obstante, las cifras de incidencia europeas son bastante menores que la nacional y las descritas en nuestro estudio. Por ejemplo, la tasa global es de 7,1 casos por cien mil habitantes; la mayor tasa se observa en Islandia (15,5 casos por 100.000 habs.) y, la menor, en Croacia (0,7 casos por 100.000 habs.); España presenta 10 casos por cien mil habitantes 8 .

En relación a la distribución por sexo, en todos los reportes y publicaciones revisados, la morbilidad en hombres es, al menos, dos veces mayor que en mujeres. En nuestro estudio se presentaron 1,7 casos de hombres por cada mujer, mientras que nacionalmente son 1,8 casos de hombres por cada mujer ${ }^{20}$. Un estudio en Italia encontró 4 casos de hombres por cada caso de mujer y en la Unión Europea (EU) se describen 8,5 casos por mujer ${ }^{8}$. Esta gran diferencia observada entre hombres y mujeres es explicada por el Centro Europeo de Prevención y Control de Enfermedades (ECDC) en base a la observación de que la mayor parte de estos hombres son jóvenes que tienen sexo con hombres $(\mathrm{HSH})^{8}$. Efectivamente, en la EU $67 \%$ de los casos reportan ser $\mathrm{HSH}^{8}$. En nuestro estudio, este porcentaje alcanzó a cerca de $15 \%$; sin embargo existió $6 \%$ de casos que no informaron su preferencia sexual, pudiendo esta cantidad ser mayor. La explicación del ECDC sobre el origen de este nuevo perfil epidemiológico radica en la introducción de terapia antiretroviral y el uso de profilaxis pre exposición (PrEP) para VIH entre $\mathrm{HSH}$, que se traduciría en conductas sero-adaptativas como: no limitar el número de parejas sexuales, tener relaciones casuales y flexibilizar el uso de preservativos ${ }^{8}$. Se origina así una falsa sensación de protección: el uso de PrEP reduce la transmisión de VIH, no así de otras ITS. En Chile, el acceso a PrEP es aún limitado, pero a futuro debe ser una variable más a considerar para el control de otras ITS.

Está descrito que presentar una ITS aumenta el riesgo de adquirir otra ${ }^{2,15}$. En efecto, la proporción de personas que presentaron co-infección con VIH en nuestro estudio fue de cerca de $10 \%$ y $20 \%$, otras ITS. En Chile la co-infección VIH alcanza a $17 \%{ }^{16}$, similar a $20 \%$ descrito para Italia ${ }^{3}$. Nuestros resultados demuestran mayor riesgo de presentar co-infección con otras ITS en hombres que en mujeres, al igual que estudios internacionales ${ }^{1,3}$.

El estudio evidencia deficiencias del sistema de atención. Primeramente, los mensajes preventivos no estarían llegando a los usuarios, a pesar de ser atendidos en una unidad exclusiva para ITS. Si bien, en la población general chilena, el uso de condón es bastante limitado (solo 10\% reporta uso de condón "siempre", según la última Encuesta Nacional de Salud ${ }^{21}$; en el grupo de usuarios estudiados esa proporción fue menor $(6,4 \%)$, siendo totalmente insuficiente para prevenir cualquier transmisión. En segundo lugar, las acciones para cortar la cadena de transmisión aparecen muy limitadas; tanto la adherencia al tratamiento, como la búsqueda y el tratamiento de contactos son insuficientes. Se espera que el equipo de salud realice estas actividades, según la normativa nacional $^{2,9}$; sin embargo, dicha normativa no establece estándares, lo cual impide realizar una evaluación de las cifras obtenidas². Tercero, el diagnóstico es tardío; cerca de la mitad de los casos se diagnostican en fase latente precoz y un tercio, en fase latente tardía; similar a lo descrito por Cáceres para Chile ${ }^{16}$. Esta situación dista bastante de lo reportado en Europa, donde la mayoría de los casos se diagnostican en fase latente precoz (37\%), 
primaria (33\%) o secundaria ( $25 \%$ ); y solo $2 \%$, en fase tardía ${ }^{8}$. Igualmente, en Italia, cerca de la mitad de los casos se diagnostican como sífilis primaria ${ }^{3}$.

Finalmente, la proporción de sífilis en mujeres gestantes fue menor que la observada en Chile el año 2017, la cual alcanzó a $23 \%{ }^{16}$. En relación a las características y resultados perinatales de la sífilis gestacional, no es posible realizar comparaciones, al no ser información contenida en los reportes oficiales. Persiste una escasa capacidad de tratar a los contactos. La alta adherencia al tratamiento en gestantes, se traduce en $70 \%$ de resultados perinatales favorables. Sin embargo, existen complicaciones perinatales como partos prematuros $y$ una muerte neonatal.

En síntesis, la sífilis sigue siendo una patología de alta morbilidad, cuya incidencia se está elevando preocupantemente entre varones jóvenes, aunque también en adolescentes de ambos sexos. El riesgo de contagio en mujeres sería mayor que en hombres, probablemente relacionado con el significativo menor uso de preservativos entre ellas. El sistema sanitario tiene muchos espacios para mejorar en cuanto a prevención de las ITS: urge promover masivamente el uso de condón; así como corregir la oportunidad de diagnóstico y controlar la transmisión de la enfermedad, optimizando el seguimiento y tratamiento de los contactos de las personas infectadas, incluyendo a embarazadas; $y$, adicionalmente, acrecentar la adherencia al tratamiento y proveer educación y consejería efectivas en el box de atención.

\section{Referencias}

1. Spiteri G, Unemo M, Mårdh O, Amato-Gauci AJ. The resurgence of syphilis in high-income countries in the 2000s: a focus on Europe. Epidemiol Infect 2019; 147: e143.

2. Santander E, Sigrid C, Primera D, Barrera A. Normas de manejo y tratamiento de las Infecciones de Transmisión Sexual (ITS)*. Primera parte Sexually Transmitted Infections: Guidelines for their diagnosis and treatment. First Part. Rev Chil Infect [Internet] 2009; 26 (2): 17490. Available from: http://www.minsal.cl/ici/guias_clinicas.html

3. Roncarati G, Fazio C, Gaspari V, Marziali G, Furlini G, Galli S, et al. Syphilis in a high-density urban area in the North of Italy. New Microbiol [Internet] 2019; 41 (2): 166-70. Available from: http://www.ncbi.nlm.nih.gov/ pubmed/31157398
4. Departamento Programa Nacional de Prevención y Control del VIH/SIDA e ITS. Plan Nacional de Prevención y Control del VIH/SIDA e ITS 2018-2019 [Internet]. Santiago de Chile: Ministerio de Salud, Gobierno de Chile; 2018. Available from: https://diprece.minsal. cl/wp-content/uploads/2019/06/2019.06.12_PLANNACIONAL-VIH-SIDA-E-ITS.pdf?forcedefault=true.

5. O’Bryrne P, MacPherson P. Syphilis. Br Med J 2019; 4159 (June): 1-11.

6. Newman L, Kamb M, Hawkes S, Gomez G, Say L, Seuc A, et al. Global Estimates of Syphilis in Pregnancy and Associated Adverse Outcomes: Analysis of Multinational Antenatal Surveillance Data. PLoS Med 2013; 10 (2).

7. Díaz J. Norma Conjunta de Prevención de la Transmisión Vertical del VIH y la sífilis: Programa Nacional de Prevención y Control de la infección por VIH/SIDA e ITS División de Prevención y Control de Enfermedades Subsecretaría de Salud Pública Ministerio de Salud. Rev Chil Infectol 2013; 30 (3): 259-302.

8. European Center for Disease Prevention and Control. Syphilis Annual Epidemiological Report for 2017 [Internet]. Vol. 393. Stockholm; 2019. Available from: https://ecdc.europa.eu/sites/portal/files/documents/ AER_for_2017-malaria.pdf.

9. Díaz J. Vigilancia epidemiológica de sífilis y gonorrea. Rev Chil Infectol. 2013;30(3):303-10.

10. Korenromp EL, Ríos C, Apolinar ALS, Caicedo S, Cuellar D, Cárdenas I, et al. Prevalence and incidence estimates for syphilis, chlamydia, gonorrhea, and congenital syphilis in Colombia, 1995-2016. Rev Panam Salud Pública 2018; 42: 1-10.

11. Carazo PH. Vigilancia epidemiológica de sífilis (A50-A53) y gonorrea (A54). Rev Chil Obstet Ginecol 2013; 78 (5): 395-402.

12. Ministerio de Salud de Chile. Norma Conjunta de Prevención de la Transmisión Vertical del VIH y la Sífilis. Santiago de Chile: Ministerio de Salud, Gobierno de Chile; 2012.

13. Garbin AJÍ, Martins RJ, Belila N de M, Exaltação SM, Garbin CAS. Reemerging diseases in Brazil: sociodemographic and epidemiological characteristics of syphilis and its under-reporting. Rev Soc Bras Med Trop 2019; 52: e20180226.

14. Benavides I. Recomendaciones para el manejo del recién nacido hijo de madre VDRL (+) y del recién nacido de madre VIH (+). Rev Pediatría Electrónica 2004; 1 (1): 32-9.

15. Hernandez I, Johnson A, Reina-Ortiz M, Rosas C, Sharma V, Teran S, et al. Syphilis and HIV/Syphilis Co-infection Among Men Who Have Sex With Men (MSM) in Ecuador. Am J Mens Health 2017; 11 (4): 823-33. 
16. Cáceres K, Martínez R. Situación epidemiológica de sífilis (CIE 10: A50-A53.9). Chile, 2016. Rev Chilena infectol 2018; 35 (3): 284-96.

17. Instituto Nacional de Estadísticas. Publicación Estadísticas Vitales [Internet]. Santiago de Chile; 2019. Available from: https://www.ine.cl/docs/default-source/demográficas-y-vitales/vitales/anuarios/anuario-2017/estadísticas-vitales-cifras-provisionales-2017.pdf?sfvrsn $=4$.

18. FONASA. Boletin_Estadistico_2015_2016. Santiago de Chile.

19. Ministerio de Salud de Chile. Instructivo Boletin Notificación De Enfermedades De Declaración Obligatoria ( Boletín E . N . O .) [Internet]. Chile; 2009 p. 1-2. Available from: https://huap.redsalud.gob.cl/ wrdprss_minsal/wp-content/uploads/2012/08/formulario_ENO.pdf.

20. Departamento de Epidemiología. Ministerio de Salud de Chile. Boletín epidemiológico trimestral sífilis, Edición Nº1 año 2019 [Internet]. Santiago de Chile; 2019. Available from: http://epi.minsal.cl/boletin-epidemiologico-trimestral-edicion1-2019/

21. Ministerio de Salud de Chile. Encuesta Nacional de Salud 2016-2017 Primeros resultados [Internet]. Santiago de Chile; 2017. Available from: http://web.minsal.cl/ wp-content/uploads/2017/11/ENS-2016-17_PRIMEROS-RESULTADOS.pdf. 